Cahiers $d u$ MONDE RUSSE

\section{Cahiers du monde russe}

Russie - Empire russe - Union soviétique et États indépendants

$48 / 4 \mid 2007$

Varia

\title{
Antonello Venturi, éd., Franco Venturi e la Russia
}

\section{Alessandro Stanziani}

\section{OpenEdition \\ Journals}

Édition électronique

URL : https://journals.openedition.org/monderusse/6128

DOI : 10.4000/monderusse. 6128

ISSN : $1777-5388$

\section{Éditeur}

Éditions de l'EHESS

\section{Édition imprimée}

Date de publication : 2 décembre 2007

Pagination : 834-836

ISBN : 978-2-7132-2148-4

ISSN : $1252-6576$

\section{Référence électronique}

Alessandro Stanziani, « Antonello Venturi, éd., Franco Venturi e la Russia », Cahiers du monde russe [En ligne], 48/4 | 2007, mis en ligne le 16 juin 2009, consulté le 03 septembre 2022. URL : http:// journals.openedition.org/monderusse/6128; DOI : https://doi.org/10.4000/monderusse.6128

Ce document a été généré automatiquement le 3 septembre 2022

Tous droits réservés 


\title{
Antonello Venturi, éd., Franco Venturi e la Russia
}

\author{
Alessandro Stanziani
}

\section{RÉFÉRENCE}

Antonello Venturi, éd., Franco Venturi e la Russia. Con documenti inediti. Milan :

Feltrinelli, 2006, $542+$ XXIX p.

1 Cet épais volume rassemble des lettres inédites tirées des archives de Franco Venturi et des contributions présentant ces mêmes documents, voire les travaux, de l'historien italien disparu en 1994. Venturi incarnait un type d'historien, aujourd'hui quasiment disparu, qui parvenait à associer une rigueur philologique et interprétative des textes avec une extension impressionnante des domaines et des aires culturelles couverts, mais aussi avec un engagement citoyen et politique. L'antifascisme, l'adhésion au socialisme se conjuguent ainsi chez lui avec un travail monumental sur l'Europe des Lumières (sept volumes de Settecento riformatore), dont le point de départ est précisément l'hypothèse que les Lumières constituent un mouvement à l'échelle européenne, duquel des pays comme l'Italie et la Russie font partie intégrante. Dans ce cadre d'analyse, la circulation des savoirs est essentielle, dans la mesure où elle permet à Venturi de remettre en discussion des hiérarchies politiques et culturelles toutes faites, comme par exemple la domination et l'exportation du modèle français. Son Europe est décentralisée et les notions de centre et de périphérie perdent beaucoup de leur sens. L'intérêt de Franco Venturi pour la Russie s'inscrit donc dans un projet plus vaste et c'est cette dimension qui permet d'inclure son œuvre parmi les sommets de l'historiographie de la seconde moitié du xx ${ }^{\mathrm{e}}$ siècle. Dès sa parution en 1952 , son analyse du populisme russe apparaît aux yeux de la plupart des historiens comme une pièce maîtresse de l'historiographie sur ce pays, tant par l'analyse que le nombre d'auteurs et de textes examinés. Ce travail, déjà extraordinaire en soi, sort dès lors du commun une fois inscrit dans un tableau encore plus vaste, à savoir l'Europe des Lumières et ses filiations, le socialisme avant tout. Cet objet intellectuel répond en quelque sorte au problème que Venturi lui-même affirme 
vouloir envisager, celui de la liberté et de la justice. De ce point de vue, il s'inscrit dans cette tradition politique et intellectuelle italienne qui, depuis Mazzini, cherche à conjuguer pensée et action (pensiero e azione). C'est pourquoi le type d'histoire qu'il pratique, même s'il peut être qualifié d'histoire des idées (au sens le plus complet du terme), vise à mettre en évidence la contribution des idées à la dynamique politique et institutionnelle.

2 L'historien de nos jours a souvent du mal à rendre pleinement compte de la richesse et de la complexité de cette œuvre. Ainsi, le choix de limiter pour ce volume l'analyse aux relations entre Franco Venturi et la Russie correspond, certes, à l'importance des matériaux et des archives concernés et répond en partie aux intérêts du fils de Venturi, Antonello, maître d'œuvre de l'ouvrage et lui aussi historien de la Russie. En même temps, ce choix est révélateur de l'état de l'historiographie actuelle, toujours prisonnière du concept d' «aires culturelles» et le plus souvent incapable de sortir des cadres " nationaux » d'analyse. Très peu d'historiens seraient capables, de nos jours, de se livrer à des analyses aussi détaillées et aussi étendues que celles de Venturi et d'y inclure à la fois la Russie, la plupart des pays d'Europe dite "orientale», l'Europe méridionale, occidentale et l'Europe du Nord, et ce en consultant la quasi-totalité des sources dans leur langue originale. Ce n'est pas un hasard si ce souffle plus large se retrouve surtout dans le magnifique essai introductif de Michael Confino et dans le chapitre de Daniela Steila sur « Franco Venturi et le populisme russe». De la même façon, le chapitre de Manuela Albertone ( Franco Venturi entre Russie et Europe») aide à comprendre l'insertion du «cas russe» dans une analyse plus large du mouvement européen des Lumières. Chacune à leur manière, ces interprétations servent en même temps de présentation à certains documents inédits de Venturi inclus dans l'ouvrage, en particulier un travail sur Ščerbatov et Novikov.

3 Les autres documents issus des archives sont les lettres personnelles et les rapports officiels que Venturi écrit lors de son séjour en Russie en tant qu'attaché culturel (1947-1950). Sa correspondance avec l'historien soviétique Lev Gordon complète la riche documentation tirée des archives Venturi.

Le premier groupe de documents est présenté par Adriano Viarengo, mais inspire aussi le travail d'Andrea Graziosi, «Nation, socialisme et cosmopolitisme. L'Union soviétique dans l'évolution de Franco Venturi ». La correspondance avec Lev Gordon est à l'origine de l'article de Fabio Bettanin, «La correspondance de Franco Venturi avec les historiens soviétiques (1947-1994)». Ces chapitres adoptent un registre différent des autres et s'interrogent sur l'adhésion de Venturi au socialisme, voire au modèle soviétique. La question reçut déjà en 1947 des réponses différentes de la part des diplomates italiens, certains convaincus de l'hostilité de Venturi envers l'URSS, d'autres de son adhésion au socialisme. Ces différends se retrouvent dans les interprétations récentes de l'historiographie italienne et jusque dans ce volume. Pour certains (Viarengo), Venturi était antisoviétique, hostile au marxisme mais proche du mouvement socialiste. Ce jugement confirme celui de Bettanin mais s'oppose à celui d'autres historiens italiens (par exemple Ettore Cinnella) qui ont par ailleurs mis l'accent sur l'adhésion de Venturi au système soviétique. Graziosi pousse encore plus loin l'analyse, finalement assez proche du jugement politique, lorsqu'il s'étonne du fait que Venturi, malgré ses innombrables lectures, se soit borné à prendre ses distances vis-à-vis de l'URSS, mais non pas du socialisme et qu'il n'ait jamais réellement compris par ailleurs les critiques que les économistes (comme Mises ou Brutskus) adressaient à la planification... 
5 En réalité, la correspondance de Venturi témoigne de la richesse de ses jugements malgré la surveillance dont elle faisait l'objet, mais aussi de la manière dont il concevait son travail. Ainsi, en tant qu'attaché, il consacre ses efforts à la constitution d'une bibliothèque russe auprès de l'Ambassade d'Italie, tandis que, le soir, il passe tout son temps à la Bibliothèque nationale.

6 Assez touchante est sa correspondance avec Gordon. Venturi tente en effet de contacter plusieurs historiens soviétiques, sans succès tant ils craignent la surveillance. Gordon, au contraire, n'hésite pas à se lancer dans une correspondance intense et émouvante dans laquelle il est question tout aussi bien d'historiographie que, de manière souvent implicite, de la situation en URSS. L'ouverture à l'époque du Dégel et la désillusion de Venturi au début des années 1970, les discussions sur l'opportunité de traduire ses travaux en russe, les orientations des uns et des autres, les difficultés linguistiques ressortent avec force de ses échanges avec Gordon.

7 Tel quel, cet ouvrage constitue un événement important, aussi bien pour l'histoire de l'historiographie que pour celle de l'URSS et de la culture européenne. Il permet de tisser des liens importants entre débat intellectuel et débat politique pour une large moitié du $\mathrm{xx}^{\mathrm{e}}$ siècle, dans un cadre où l'expérience soviétique demeure irréductiblement européenne. Les relations entre historiens, malgré le rideau de fer (et au-delà), la construction d'un objet historiographique, le populisme et les Lumières, dans un contexte historique marqué par l'opposition et la fragmentation de l'espace européen tous ces éléments sont au cœur de l'œuvre de Venturi et de cet ouvrage et en rendent hautement souhaitable une diffusion rapide dans d'autres langues. 